\title{
Room at the Bottom is Growing...
}

In February 1960, Richard Feynman published his now-legendary article entitled "There's Plenty of Room at the Bottom" in Engineering and Science magazine issuing, as he put it, an invitation to enter a new field - that of nanometer-scale science and technology. In addition to making a number of remarkably prescient observations, Feynman also set forth two challenges, replete with prizes: to construct a working electric motor with linear dimensions of $1 / 64$ of an inch and to shrink a page of readable text by $1 / 25,000$. An illustration of the practical difficulties in scaling dimensions of physical objects by orders of magnitude is that the first challenge was met by 1962, but it was not until 1987 that a group at Stanford University accomplished the second, using electron beam lithography. At least in hindsight, the pressures for miniaturization have been relentless. The "top down" approach exemplified by integrated circuit technology has enabled reductions by many orders of magnitude in size, and now the power of "bottom up" approaches that exploit knowledge of chemically and biologically directed assembly techniques is becoming apparent. Where "top down" meets "bottom up," we can anticipate a flourish of scientific development and engineering possibilities. These general trends have also been greatly abetted by science breakthroughs, such as the demonstration of vacuum tunneling that has led to the scanning tunneling microscope, and density functional theory computations that have produced tremendous theoretical insights about atomic-scale structure. But even before any of the wonderful tools of nanoscale science and technology existed, Feynman understood the vast potential of the nanoscale, "It is a staggeringly small world that is below. In the year 2000, when they look back at this age, they will wonder why it was not until the year 1960 that anybody began seriously to move in this direction."

Where did Feynman's enthusiasm take us?

On January 21, 2000, standing in front of an image of the Western hemisphere written in gold atoms, President Bill Clinton announced the National Nanotechnology Initiative (NNI), a U.S. federal budget proposal for the 2001 fiscal year slated to invest hundreds of millions of new dollars in research programs in nanoscience and engineering (see the MRS website < www. mrs.org/pa/nanotech $>$ for details and

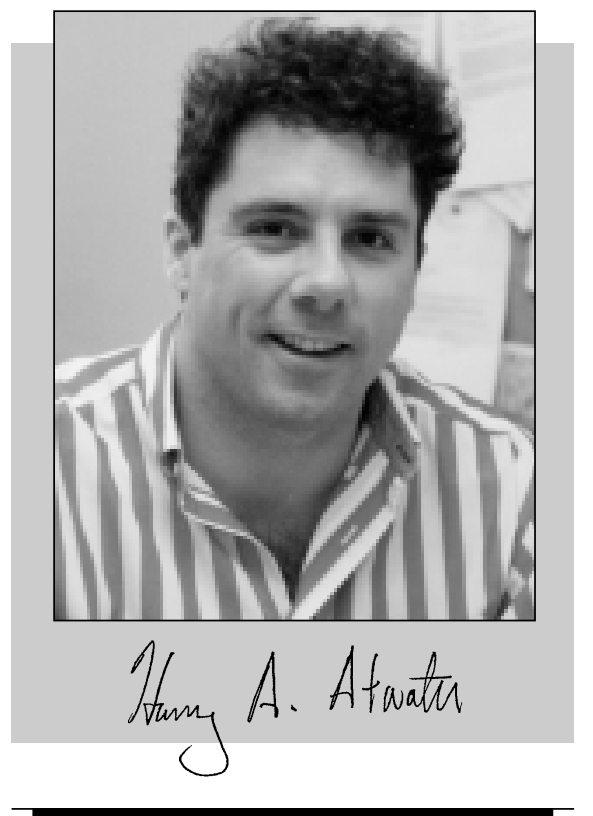

If it comes anywhere close to full fruition, the [National Nanotechnology Initiative] is likely to have an intellectual, financial, and cultural impact on almost every MRS member, including those outside the United States.

links). In his State of the Union address to Congress delivered a week after the nanotechnology announcement, the President remarked, in reference to nanoscale technology, "Soon researchers will bring us... materials 10 times stronger than steel at a fraction of the weight; and-this is unbelievable to me-molecular computers the size of a tear drop with the power of today's fastest supercomputers." I found it no less than amazing to hear such words being spoken in the U.S. Capitol by a nonscientist President to a nonscientifically oriented Congress and largely nonscientific public. Clearly nanoscience and engineering have the power to inspire the public imagination. So while to us materials researchers everything has been "nanothis" and "nano-that" for some time, it is worth reflecting for a moment that we are a group of professionals who are in the everyday habit of projecting our scientific reach and grasp to a scale one one-billionth the size of our own hands. And that's pretty cool.

If it comes anywhere close to full fruition, the NNI is likely to have an intellectual, financial, and cultural impact on almost every MRS member, including those outside the United States. Since the impact is likely to be of net benefit to MRS members, MRS has voiced its support for the National Nanotechnology Initiative, and was the first professional society to do so. Many members of the Society in the United States will want to make their views about it known to their elected Congressional representatives in the upcoming months, and those outside the United States will probably want to communicate the Initiative's scope and possible impact to elected officials in their nations as well.

However, a significant part of the public and political enthusiasm currently enjoyed by nanotechnology has less to do with the intrinsic desire of politicians to support materials researchers, and more to do with the considerable expectation that a countless array of communications gadgets, drugs, energy storage devices, medical procedures, and such will emanate from NNI. MRS members have a very important educational role to play in addressing the issues raised by this expectation. Foremost, we must do all that we can to generate optimistic but realistic expectations for the potential of nanoscale science and technology. We must obviously build upon the incipient enthusiasm created by the President's words and the ideas of the drafters of the Initiative. We must also deliver with honesty and candor the message that while research in nanotechnology has enormous potential overall, any given research project is likely to be very risky. Most such projects will fail. That they are likely to fail is an indication that they were positioned with the appropriate level of risk and reward. A few projects will likely succeed spectacularly, and they will make all the difference in terms of satisfying societal expectations. There may or may not be direct correlations between the initial intent of the "successful" research projects and their eventual applications. Although President Clinton indicated in his speech that investments in nanotechnology might easily take a decade or more to bear fruit, others having a say in setting budget priorities may be less patient or more demanding of specific applications. We as researchers are very accustomed to 
the notion that risk is part of the research enterprise, but even in a time of budgetary bounty in the United States, U.S. lawmakers and citizens will not be automatically receptive to the notion that failure is built into research.

Another important educational message that MRS members need to carry forth to the public is how close nanotechnology is to every one of us. The scientific principles and observations that are the foundation for nanotechnology are not arcane, abstract, unseen, and unknowable by the public. A powerful demonstration of this is that we can see atoms. The beautiful, atomically resolved images of surfaces, interfaces, and artificially created structures are proof that observation and engineering intervention on the nanoscale is real. Yet nanoscience principles are manifest in our world in the wear of automobile tires, the color of paint, or the brightness of a traffic light.

The NNI has much to offer to materials researchers. However, it is our obligation to convey what nanotechnology has to offer to everyone else. Thirty years before Feynman's observations and almost 70 years before Clinton's announcement, Albert Einstein in 1931 remarked, "Never forget this, in the midst of your diagrams and equations: concern for man himself and his fate must always form the chief interest of all technical endeavors." One could hope that such understanding would be the real legacy of the National Nanotechnology Initiative.

HARRY A. ATWATER 2000 MRS President haa@daedalus.caltech.edu

\section{Letters to the Editor}

\section{NIST's Contribution to Neutron Scattering in Materials Research}

\section{To the Editor:}

I am writing to comment on the December 1999 issue of MRS Bulletin. I was disappointed to observe that in an issue dedicated to neutron scattering in materials research, there was no mention of the NIST Center for Neutron Research (NCNR). The NCNR is operated as a national user facility, and it serves more users than all other U.S. neutron sources combined. The facility is located in the Materials Science and Engineering Laboratory of the National Institute of Standards and Technology at the Gaithersburg, MD site. It is the only U.S. facility offering world-class capabilities for both thermal and cold neutron research, with a fully developed cold neutron guide hall and instrumentation.

Much of the research at the NCNR is in the area of materials science, including strong programs in polymer science, highly correlated electron systems, ceramics, measurements of residual stress, powder diffraction, and other areas, including the rapidly growing area of neutron reflectivity (which was barely mentioned). In particular, the NCNR is heavily involved (both for in-house research and by outside users) in the area of soft materials (i.e., polymers, comlex fluids, biomaterials, gels...). In fact, in recent years six major prizes have been awarded for work done largely at the NCNR in the general area of soft matter and materials research.

While we at NIST were happy to see the publicity given to the use of neutron techniques, we believe that general reviews in MRS Bulletin should be less parochial than this one was.

J. Michael Rowe

Director, NIST Center for Neutron Research

Response:

As we noted in our introduction it was not our intent to provide comprehensive reviews of the fields described in the short articles contained in the December 1999 issue of MRS Bulletin. Rather, the aim was to give a brief introduction to selected applications of neutrons to problems of interest to the materials science community. If brevity has led to the appearance of a neglect of the important contributions of the NIST facility, then we apologize; that was not the intent. Indeed, NIST has made important contributions to neutron scattering across the range of science addressed in the Bulletin articles. For example, one area of particular significance is in studies of biomolecular materials carried out at NIST using neutron reflectometry highlighted in the article by J.K. Blasie and P. Timmins [page 40].

Thom Mason, Guest Editor Oak Ridge National Laboratory and

Andrew Taylor, Guest Editor Rutherford Appleton Laboratory

\section{Access Materials Gateway on the Materials Research Society Web Site}

Professional Societies
Materials related associations and organizations
Academic Departments
University departments and research centers
Government Organizations
Including national laboratories
Materials Information
Including W eb-based databases

Including W eb-based databases
Publications

Materials related journals and other publications

Educational Resources

Resources on the W eb for materials related educational resources

Companies

Materials industries

USENET Newsgroups

Materials research related discussion groups
Materials Related Meetings

Upcoming meetings, conferences, workshops and symposia of interest to materials researchers

Materials Related News

Materials related news culled from various sources

What is Materials Research?

General information about the field of materials including hyperlinks to educational resources on the $\mathrm{W}$ eb

National Nanotechnology Initiative

Information relevant to materials researchers

w w w . m rs.org/gateway/ 